\title{
El diálogo paradigmático en Educación Social: la simulación como un modo de enseñar y aprender en las aulas universitarias
}

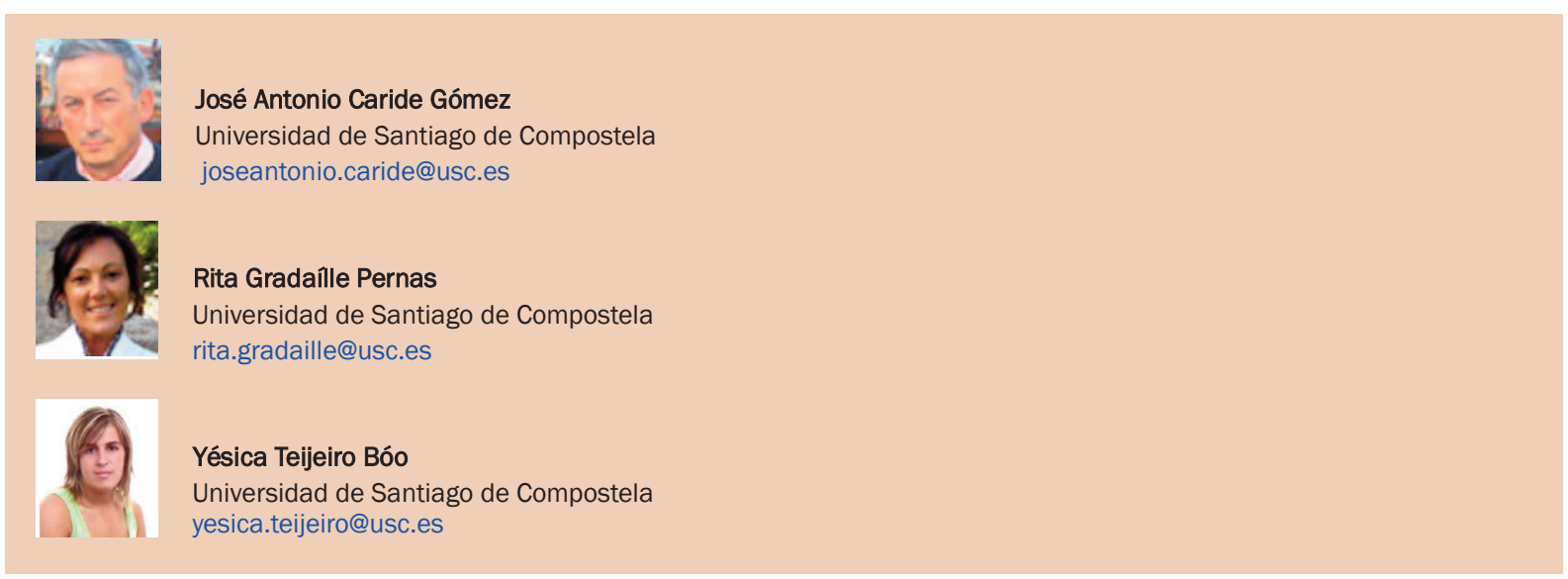

|Fecha presentación: 10/10/2012 | Aceptación: 08/11/2012 |Publicación: 20/12/2012

\begin{abstract}
Resumen
El texto presenta una práctica de simulación en el aula, realizada con estudiantes de 'Pedagogía Social' en la Diplomatura de Educación Social y en la Licenciatura de Pedagogía (ahora Grados), en la Universidad de Santiago de Compostela.

Con un enfoque didáctico innovador, el alumnado adquiere protagonismo en los procesos de enseñanza-aprendizaje, y sus planteamientos metodológicos se proyectan en un 'diálogo' que toma como referencia los paradigmas (tecnológico, hermenéutico-interpretativo y socio-crítico) a los que suele remitirse la Pedagogía-Educación Social.

Mediante un juego de roles colectivo, las teorías comprometen las miradas de quienes las 'representan', desarrollando los conocimientos de un modo estimable.
\end{abstract}

Palabras clave: pedagogía Social, simulación, paradigmas, innovación didáctica, universidad

\section{Resum}

El text presenta d'una pràctica de simulació a l'aula, fet amb els alumnes "Pedagogia Social" en la diplomatura en Educació Social i grau de Pedagogia (graus d'ara), a la Universitat de Santiago de Compostela.

Amb un enfocament didàctic innovador, estudiants adquireixen un protagonisme en el procés d'aprenentatge, i seus plantejaments metodològics es projecta sobre un "diàleg", que tenen com a seu referència els paradigmes (Tecnològics, Hermenéutico-interpretativo i Socio-Critique) d'aquells que normalment es refereix a la Social.

Generant un joc de rol col.lectiu, les teories es comprometen les mirades dels que representar-los, desenvolupar el coneixement d'una manera estimable.

Paraules clau: pedagogia social, la simulació, els paradigmes, la innovació docent, universitat

\section{Abstract}

The paper presents a simulation exercise in the classroom, realized -in the last years- with students who have studied the subject of 'Social Pedagogy' in the Diploma in Social Education and the Bachelor of Education (now Degrees) at the University of Santiago de Compostela.

With an innovative teaching approach, in which students acquire a role in the teaching-learning process, projecting their methodological approaches in a 'dialog' that takes as reference the paradigms (technological, interpretative and socio-critical) usually refer to the Social Education.

By means of a collective game of roles, the theories compromise the looks of those who 'represent' them, developing the knowledge of an estimable way.

Keywords: social pedagogy, simulation, paradigms, teaching innovation, university 


\section{Introducción}

Suele decirse que la simulación consiste en la capacidad de representar algo, fingiendo o imitando lo que no es. Así se la define en los diccionarios, presionados por la exigencia -más o menos explícita- de reducir a pocas palabras realidades o hechos caracterizados por una gran complejidad. Afortunadamente, la creatividad humana -y las circunstancias en las que inscribimos nuestra capacidad para imaginar y activar nuevos significados en las palabras- permiten que el acto de simular se proyecte en un diversificado campo semántico, especialmente sugerente para el quehacer educativo y los procesos de enseñanza-aprendizaje.

Entre otros, le debemos a Bruner (1969) la posibilidad de que las utilidades prácticas de la simulación se vean reflejadas en el aprendizaje por descubrimiento, motivando a los educadores y a los estudiantes para que aprovechen sus vivencias como un recurso formativo de amplios recorridos pedagógicos y sociales, en las aulas y en muchos otros contextos académicos, institucionales, profesionales, etc. Una opción, sin duda, estimulante para "aprender a experimentar y comprender situaciones cambiantes" (Matas 2003: 282), en las que el sentido lúdico, la reflexión y el debatediálogo colectivos, la toma de decisiones, la aplicación de conocimientos, o el trabajo cooperativo..., les conceden un especial protagonismo a quienes personifican -individual y grupalmente- este tipo de dinámicas.

Con esta perspectiva, concebimos las simulaciones como prácticas totales, que se pueden emplear en distintos niveles y etapas del sistema educativo (desde la Educación Infantil hasta la Educación de Adultos, pasando por la Formación Profesional o las enseñanzas universitarias), en programas de educación social, animación sociocultural, formación ocupacional, etc. Al fin y al cabo, se trata de un tipo de experiencia-vivencia pedagógica abierta a las múltiples contingencias de enseñanza y aprendizaje que ofrecen las realidades sociales, desde el estudio de un caso hasta un juego de roles (interpretación), ya sea en un escenario espacial y temporalmente delimitado, y/o a través de la creciente y cada vez más sofisticada gama de dispositivos audiovisuales, electrónicos y virtuales. Como recuerdan Arias-Aranda (2008), no debe obviarse que desde los primeros años cincuenta del pasado siglo, los juegos de simulación han ido mejorando a medida que la tecnología del hardware y del software también evolucionan, de modo que en la actualidad los modernos equipos de electrónicos (ordenadores, tablets, móviles, etc.) permiten simulaciones en cualquier ámbito del aprendizaje, sobre todo en aquellos que se vinculan con la planificación o la dirección estratégica, el análisis y la predicción-anticipación de procesos decisionales, dinámica de sistemas, etc.

\section{La simulación: una práctica educativa orientada a diálogo y al aprendizaje compartido}

En las Universidades las simulaciones constituyen una excelente herramienta metodológica para aproximar los procesos de enseñanza-aprendizaje a problemas o realidades de cierta complejidad, que los métodos más convencionales difícilmente pueden afrontar. No, al menos, con la visión holística e integradora que se requiere para el desarrollo cognitivo, emocional e instrumental que se explicitan en muchas de las competencias -referidas al saber, el saber hacer y el saber ser- que se declaran en las motivaciones de los Planes de Estudio, en las guías docentes o en las programaciones que se les ofertan a los estudiantes para que pue- dan graduarse. En este sentido, como señalan Graemi, Baena y Mihai (2010), la puesta en práctica de casos reales en clase y del desarrollo de problemas en el medio académico, debe permitir que los alumnos/as no sólo conozcan en profundidad los contenidos de las asignaturas, sino también su aplicación en entornos laborales cambiantes y complejos; un logro que la simulación favorece, en sus distintas modalidades (estudio de casos, aprendizaje basado en problemas, juego de roles, videojuegos, etc.), dentro y fuera del aula.

Entre otras potencialidades formativas nos quedamos con las que, acertadamente, resume Jesús Valverde (2010: 88), al indicar las contribuciones de la simulación educativa al aprendizaje de la Historia, a pesar de su tendencia a considerar dicha simulación como una práctica que, haciendo uso del ordenador, "permite a los alumnos manipular sistemas complejos, modificando el valor de las variables que los afectan y observando los resultados con el fin de construir su propio conocimiento". Este mismo autor, en una aportación previa, indica que una simulación es adecuada para favorecer la comprensión de ideas y conceptos abstractos, así como para el aprendizaje de estrategias o la adquisición de una visión global de una realidad. Una buena simulación es capaz de generar pensamientos y sentimientos profundos en sus participantes (Valverde 2008). De ahí que adaptemos sus argumentos a nuestra propia experiencia en el desarrollo académico de la Pedagogía Social: una práctica de simulación en la que, sin utilizar el ordenador -más allá de visionar un documental, de mostrar una presentación en power point o de conducir una audición musical- y apelando al diálogo paradigmático en la Educación Social, los estudiantes son el principal soporte de los procesos de enseñanza-aprendizaje que se promueven, a partir de la lectura de los textos que les han sido entregados y de las dinámicas interactivas que, secuencialmente, se llevan a cabo contando con su activa participación. En este sentido, los objetivos que se pretenden alcanzar son:

- Contribuir a mejorar la comprensión de ideas y conceptos abstractos (los paradigmas y los modelos de racionalidad teórica); promover una visión global de una determinada realidad; en nuestro caso, las tensiones riqueza-pobreza, interrogándonos acerca de qué significa ser pobre en la sociedad actual, quienes son las personas pobres del nuevo milenio y qué podrá o deberá hacer por ellos la Pedagogía-Educación Social;

- Poner en juego las propias decisiones, identificando y resolviendo problemas o situaciones, planteando y procurando dar respuesta a cuestiones de naturaleza y alcance político, social, económico, ético, pedagógico, etc.; - Circular por el espacio y el tiempo, según las necesidades educativas del momento presente, sobre todo cuando resulta imposible tener una experiencia directa de sus circunstancias, viviendo las realidades de la gente como si nos concerniesen individual y colectivamente, ya sea como ciudadanos o como profesionales de la acción-intervención social;

- Conocer y experimentar en primera persona el impacto de una acción o propuesta, no solo en materia de políticas sociales, educativas, culturales, sino también de la práctica profesional de quienes están en su formación inicial.

Interesa poner énfasis en que, además de presentar o afrontar un tema-problema, a la que se aportan datos reales 
a través de documentos escritos y audiovisuales, son los propios estudiantes los que deben interiorizar y socializarse en el rol específico que les corresponde asumir-defender ante los demás. Aludimos a una identidad que se les atribuye azarosamente, con la que pasan a ser miembros de una de las tres comunidades científicas y profesionales que articulan los modelos paradigmáticos -teorías- a las que son adscritos (tecnológico, hermenéutico-interpretativo y socio-crítico), en las cuales y desde las cuales han de argumentar sus respectivos posicionamientos acerca del tema-problema objeto de estudio: Educación social, desarrollo y pobreza: perspectivas en el afrontamiento de la exclusión y la marginación social.

Orientamos la dinámica apelando al pluralismo teórico como un argumento epistemológico sustancial para el quehacer pedagógico-social (Caride, 2003), resumiendo el discurso que se presenta a los estudiantes en los siguientes enunciados:

1. Etimológicamente, la palabra teoría (theoria) alude a un modo de mirar al mundo y sus circunstancias, juiciosa y serenamente.

Ese fue su sentido filosófico originario. El paso del tiempo lo reconvirtió en un amplio y variado elenco de significados, en los que se articulan diversas formas de explicar e interpretar las realidades, incluso de transformarlas, tomando en consideración los aportes derivados del conocimiento científico (principios, leyes, teoremas, modelos, etc.) y de sus aplicaciones o intervenciones en el curso de los hechos, y que a menudo identificamos como práctica.

2. La teoría, en verdad, son teorías, en las que se expresan diferentes opciones de mirar, indagar, comprender, explicar, saber, proponer, intervenir, juzgar..., realidades, en lo que son y/o deberían ser.

De ahí su pluralidad. De ahí también la permanente necesidad de tomar postura, de interesarse en lo que se parecen y en lo que se distinguen..., o de valorar la congruencia que existe entre lo que se dice y lo que se hace en su nombre. Al fin y al cabo, siempre nos habita una teoría, implícita o explícitamente, por lo que es importante saberse partícipe de unos o de otros modos de pensar y actuar como pedagogos-educadores sociales. No sólo para sustentar la reflexión y la acción socioeducativa en unos determinados modelos de racionalidad teórica -a los que podemos identificar como paradigmas- sino también para darles la oportunidad de dialogar entre ellos, de evitar el pensamiento único, de concertar pareceres, de eludir los dogmatismos, el eclecticismo y el relativismo.

El saber que se crea o expresa a través de la Pedagogía Social y de la Educación Social debe participar plenamente del debate paradigmático, propiciando una permanente revisión de los valores, conocimientos y prácticas sociales en los que se sustentan. Con este propósito, presentamos a los estudiantes los modelos paradigmáticos más significativos, tratando de acentuar las diferencias que existen entre ellos. Seguimos, en este sentido, los planteamientos de Habermas (1982) para definir la teoría crítica y especificar de qué manera se relaciona con otras formas de conocimiento, mostrando algunas de las clasificaciones tipológicas de lo que ha dado en llamarse el discurso o esquema de las tres perspectivas, en alusión a las tres formas de conocimiento que el filósofo alemán maneja en sus primeras obras: el empírico-analítico, la hermenéutica y la teoría crítica. Adaptando sus denominaciones, aún a riesgo de simplificar, cada uno de ellos se describe con los siguientes perfiles:

a) Paradigma tecnológico (empírico-analítico): concurrente con la idea positiva del conocimiento, sostiene que existe unidad de método entre las Ciencias Naturales y las Ciencias Sociales; éstas tendrían que buscar generalizaciones permanentes similares a leyes, rechazando explicaciones que se remitan a estados individuales subjetivos. Existe predilección por la cuantificación, el control técnico y la predicción a través del conocimiento nomológico. Los problemas han de encontrar sus soluciones a través de procedimientos técnicoracionales, con un proceso que suele adoptar una lógica lineal, cuyas fases se articulan en secuencias que conllevan la definición del problema, la revisión de la literatura, la formulación de las hipótesis, las operaciones de instrumentación (recogida de información e intervención conforme al diseño, etc.), obtención de resultados, evaluación, presentación de conclusiones, retroalimentación. El investigador enfatiza el método, busca la verdad última de la realidad, pone énfasis en la acción instrumental, en la eficacia, eficiencia y la racionalidad del proceso y de sus logros asociados.

b) Paradigma hermenéutico (interpretativo-simbólico): subraya el protagonismo que tiene la comprensión humana en el conocimiento, considerando que las realidades sociales se configuran a través de interacciones simbólicas y pautas de comportamiento que ponen en juego las personas y los grupos sociales. La hermenéutica pretende comprender dentro de un contexto de significado intersubjetivo, postulando la existencia de una diferencia cualitativa entre los métodos de las Ciencias Sociales y los de las Naturales. Su desarrollo metodológico está abierto a la invención, la creatividad, el descubrimiento y la interpretación contextual, en un proceso circular que parte de una experiencia o situación que se trata de interpretar con los diversos puntos de vista de los implicados. De ahí que se ponga énfasis en la importancia del lenguaje, de la interacción y de la comunicación.

c) Paradigma socio-crítico (dialéctico-político): se asocia a la indagación crítica, dialéctica y transformadora, otorgándole especial protagonismo a las relaciones asimétricas o de poder que se dan en la sociedad. Respaldado por el conocimiento socio-histórico y la articulación de los paradigmas materialista e interpretativo pretende desvelar las intenciones ocultas de las ideologías y la sociedad desigual, aspirando a la emancipación de las personas y de las comunidades. El cuestionamiento de las distorsiones que se producen en la comunicación social y en las ideologías, revelando los vínculos que ambas tienen con el poder, refuerza la vocación y el compromiso político de esta opción paradigmática, caracterizada por una decidida voluntad de poner los logros del conocimiento y de la acción social a favor del cambio y la transformación social.

Tratando de favorecer su comprensión, así como la naturaleza y el alcance del diálogo al que son convocados, se les presenta a los estudiantes una representación gráfica (Figura 1 y Anexo A) en la que se muestran estos perfiles de un modo esquemático, reflejando aspectos como: la relación te- 


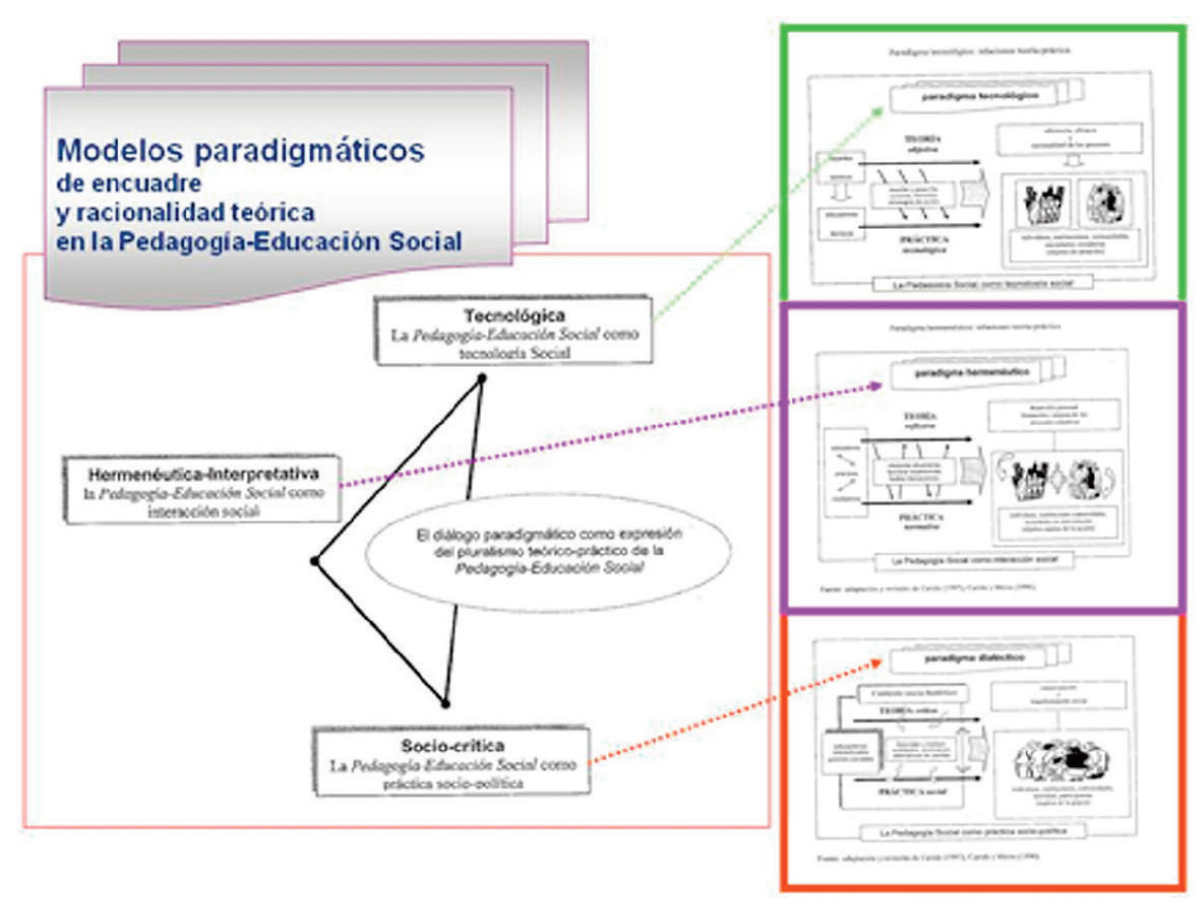

Figura 1. El diálogo paradigmático como expresión del pluralismo teórico en la Pedagogía-Educación Social oría-práctica, el tipo de conocimiento e intervención que proponen, los objetivos y las finalidades que pretenden conseguir, el rol de los sujetos y de la sociedad, etc. (Ver Figura 1).

\section{La pobreza y la exclusión social como texto-pretexto pedagógico}

Advirtiendo a los estudiantes que ninguna opción paradigmática es indemne al poder de las ideologías y a la confrontación de valores, pretendemos que sean plenamente partícipes (simuladamente, en las razones y en los sentimientos) de un tema-problema concreto, definido por las condiciones en las que se inscriben la pobreza y las desigualdades sociales en la sociedad contemporánea, tanto en los planos antropológico como psicológico, sociológico, económico, político, histórico o estrictamente vivencial. Para ello, la simulación -a modo de un juego de roles individuales y colectivos- se trata de que puedan posicionarse y dialogar sobre las percepciones, valoraciones, análisis, soluciones, alternativas, etc., que se estima sugieren cada uno de los modelos paradigmáticos: aquel al que son adscritos, cuyos argumentos defienden y desde el que dialogan -como un exponente más del pluralismo teórico y práctico de la Pedagogía Social y de la Educación Social- con los demás, si cabe llegando a algún tipo de consenso. Un diálogo articulado en torno a tres ejes temáticos, a los que se asocian otras tantas preguntas, que sintetizamos en:

a) El problema de la pobreza y sus diagnósticos en la sociedad del nuevo milenio: ¿Qué es la pobreza, quiénes son las personas pobres?

b) Las soluciones políticas y sociales: ¿Qué se puede y debe hacer para luchar contra la pobreza?

c) La Pedagogía-Educación Social ante la pobreza y la exclusión social: ¿Cómo educar ante y/o en las situaciones de pobreza?

En las argumentaciones acerca de la lucha contra la pobreza y la exclusión social se insiste en mostrar que se trata de un problema global de enormes proporciones, que afecta a casi 3.000 millones de personas en todo el mundo, con referencias explícitas a su situación en la Unión Europea y en España. Para ello, con el propósito de ejemplificar la problemática objeto de estudio e intervención socioeducativa, además de un dossier documental -con textos e informes en distintos formatos (artículos y capítulos de libros, ensayos breves, noticias de prensa, etc.)-, se les muestran textos e ilustraciones a través de una presentación multimedia, en algunos casos posibilitando la inserción de audiciones o documentales. En concreto:

- El que permite leer y escuchar la interpretación por Joan Manuel Serrat de su tema Disculpe el señor, incluido en su grabación Utopía (1992) [http://www.youtube.com/watch?v=w8BnbJNP_CI].

- La lectura colectiva de varios textos que responden al título común La voz de los pobres; Pobrezas, de Eduardo Galeano, publicado en su libro Patas arriba: la escuela del mundo al revés, editado por Siglo XXI, Madrid, en 2002; un texto del que es autor Ryszard Kapuscinski, extraído del libro Los cínicos no sirven para este oficio, editado por Anagrama, Madrid, en 2003; otro texto, breve, de Federico Mayor Zaragoza, extraído del libro Un mundo nuevo, Círculo de Lectores-UNESCO, Barcelona, en 2000.

- La presentación de varios documentales, que en los últimos años toman como referencia Vivir en la calle (Tele5, año 2000, actualmente sin enlace en internet); En el umbral (Televisión Española_2 en el programa Documentos TV, http://tu.tv/videos/en-el-umbraldocumentos-tv-20-anos); y Dueños de nada (emitido en Documanía-Canal+,

en 2003 , http://vimeo.com/3152397).

En alguno de estos reportajes se muestra la crudeza de la pobreza y de los rostros en los que se ha instalado de un modo crónico, con una visión macro y micro social. En las fichas de trabajo que se les entregan a los alumnos y alumnas se refleja textualmente en los testimonios de sus prota- 
gonistas: "no se trata de explotar el sensacionalismo, sino de sensibilizar. Llamar la atención sobre la pobreza que se vive a nuestro lado y que tiene caras distintas"; "Todos ellos explican la vergüenza que les da pedir, la fuerza con que tienen que sobreponerse a la inevitable depresión de verse así, o cómo comprueban que lo más duro en la calle es la indiferencia o el desprecio de quienes pasan a su lado sin inmutarse"; "Todos ellos son ciudadanos de la Unión Europea, esa parte privilegiada del 10\% de la humanidad que acumula el 70\% de la riqueza. Una riqueza que a los protagonistas de 'En el umbral' se les resiste. No son marginados pero se sienten al margen. Una sensación que comparten con miles de ciudadanos que, sin embargo, prefieren ocultarse"; o, finalmente, "los colectivos más vulnerables son: mujeres solas con hijos, parados de larga duración, ancianos e inmigrantes. La Comisión Europea reconoce que aunque la pobreza no campa por Europa como en otros continentes, en medio de la prosperidad las desigualdades alcanzan una magnitud considerable". Hoy, diez años después, todavía es peor en Grecia, Portugal, España, Italia, etc.

Como hemos venido señalando, la dinámica de la simulación se vincula al desarrollo temático de la asignatura de Pedagogía Social, que los estudiantes de la Facultad de Ciencias de la Educación de la Universidad de Santiago de Compostela han de cursar obligatoriamente en los planes de estudio de Educación Social y Pedagogía, aunque en momentos diferentes de su formación. Al respecto, decir que suscribimos la necesidad de que las metodologías de enseñanza-aprendizaje en las Universidades sean generadoras de saberes y vivencias innovadoras, coherentes con los intereses y expectativas de los estudiantes y de la disciplina que se enseña, promoviendo "situaciones de aprendizaje formativas y transformadoras en las que los participantes sientan un compromiso con el estudio y adquieran actitudes adecuadas a los nuevos retos de su futura profesión" (Medina 2001: 192). Una misión que la educación superior difícilmente podrá satisfacer si en sus actuaciones docentes, investigadoras, de extensión cultural, etc. no asume -con toda la carga ética y cívica que ello implica- las responsabilidades que, como instituciones, les es exigible, de formas diversas y en contextos diferentes (Martínez 2008). La pobreza y la exclusión social son, en este sentido, un texto-pretexto pedagógico ante el que no podemos inhibirnos ni mostrarnos indiferentes.

\section{Un proceso metodológico innovador, desde su programación hasta la evaluación}

La simulación, tal y como la hemos diseñado y aplicado combina el estudios de casos y el juego de roles; pues además de presentar una situación-problema por medio de una selección de materiales con datos reales, audiciones y documentos audiovisuales, requiere que cada participante adopte una identidad acorde con el modelo paradigmático al que estará adscrito, desde el inicio de la dinámica hasta su conclusión. Siendo así, la metodología que se propone para la realización de la simulación permite conocer las reacciones de los estudiantes ante diferentes formas de analizar e interpretar las realidades sociales, con miradas distintas a las que adopta cotidianamente, dotándose de argumentos que le permitan defender su modelo paradigmático ante los demás (con toda la convicción que se requiere en un juego de roles, aunque esté muy lejos de coincidir con sus modos de ser y de pensar), en un diálogo del que también se esperan puntos de encuentro y de consenso.

Atendiendo a las diferentes etapas que ha de seguir toda práctica de simulación, su desarrollo metodológico en el aula respondió básicamente al siguiente esquema: 1) presentación de la dinámica de simulación y descripción de sus planteamientos metodológicos; 2) adscripción de forma aleatoria de los miembros del grupo a uno de los tres modelos paradigmáticos; 3) presentación del tema-problema objeto de estudio y de la documentación que permitirá su desarrollo (de un lado, textos cuyo contenido alude al pluralismo teórico en la Pedagogía Social y la Educación Social; de otro, materiales que les permitan aproximarse a distintas circunstancias sobre los problemas del desarrollo desigual, la pobreza y la exclusión social); 4) desarrollo de las distintas sesiones (grupos completos e intermedios o pequeños, constituidos en función de los tres modelos paradigmáticos); 5) evaluación de la dinámica.

El número de participantes se determina en función de los estudiantes que asisten asiduamente a clase (entre un mínimo de 40 y un máximo de 50, según los cursos), repartidos aleatoriamente en tres subgrupos, que se identificarán con los tres modelos paradigmáticos que ya se han mencionado: tecnológico, hermenéutico-interpretativo y socio-crítico. Cada subgrupo nombrará un moderador y un portavoz, que desempeñarán roles claramente diferenciados durante las sesiones de debate en pequeño y gran grupo; correspondiéndole al primero las tareas de pautar los tiempos y las intervenciones así como animar y motivar la participación de todos los miembros del equipo; mientras que al segundo le corresponde la función de sintetizar y explicitar los argumentos de los que hará uso el grupo. Estas mismas funciones se trasladarán al debate colectivo en el que se integran los tres enfoques teóricos, que tendrá lugar en la última sesión de clase que se programe a tales efectos; y en el que los demás integrantes de cada grupo podrán intervenir, posicionarse y argumentar en un diálogo abierto una vez que el portavoz explicite los posicionamientos del modelo paradigmático que representan respecto del tema objeto de análisis. De este modo, cada alumno vivencia en primera persona el rol que le correspondería como tecnológico, hermenéuticointerpretativo y socio-crítico. Se prevé, en un futuro, realizar pequeñas grabaciones de las actividades con los estudiantes y que puedan estar disponibles en el repositorio de buenas prácticas de la USC u otras aplicaciones que lo permitan, con el fin de orientar a futuras promociones académicas y a la comunidad científica. La actividad, a realizar en el aula, y al margen del trabajo que de forma autónoma realicen los alumnos/as (individual o grupalmente), requiere una duración mínima de 4 horas y media, distribuidas en tres sesiones de hora y media cada una, siendo factible prolongarla más allá de las 10 o las 12 horas. De cómo se va conformando y secuenciando el desarrollo de la simulación dan cuenta las Figuras 2, 3 y 4.

Para profundizar en el conocimiento de las temáticas en torno a las que se construye la simulación, los docentes seleccionan una serie de lecturas (de las que ya se ha informado y a las que podrán acceder los estudiantes, ya sea en la fotocopiadora de la Facultad, o a través de la plataforma virtual de la Universidad de Santiago de Compostela), que deben ser consultadas por los participantes en la simulación antes de que se inicie, pues constituyen el soporte teórico esencial para fundamentar sus contribuciones al grupo del que forman parte y al conjunto del debate-diálogo paradigmático (de cada una de las secuencias). 
Simulación "Educación Social, desarrollo y pobreza" - Pedagogía Social

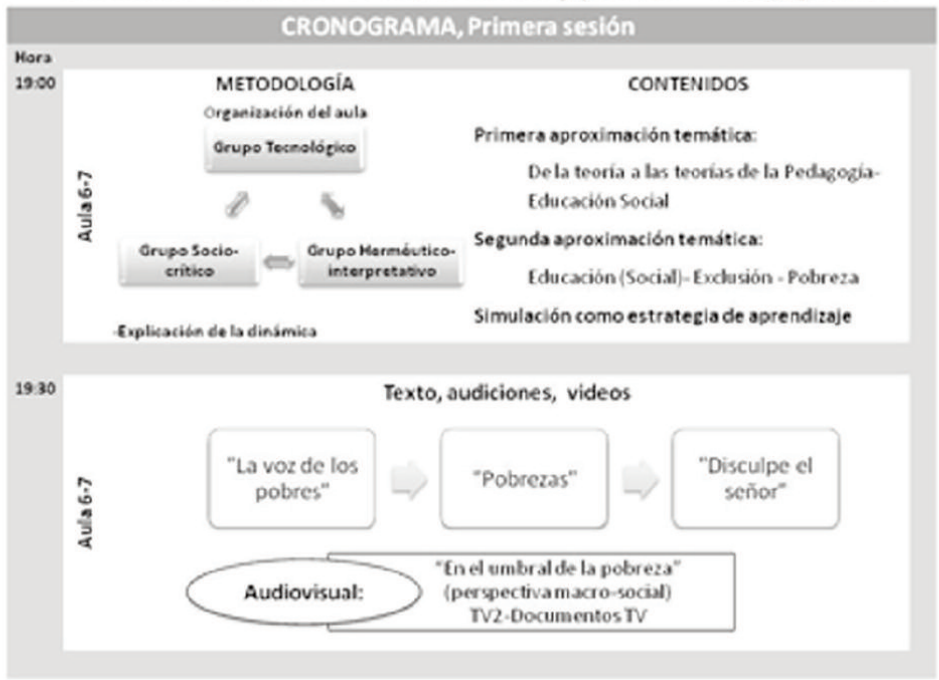

Simulación "Educación Social, desarrollo y pobreza" - Pedagogía Social

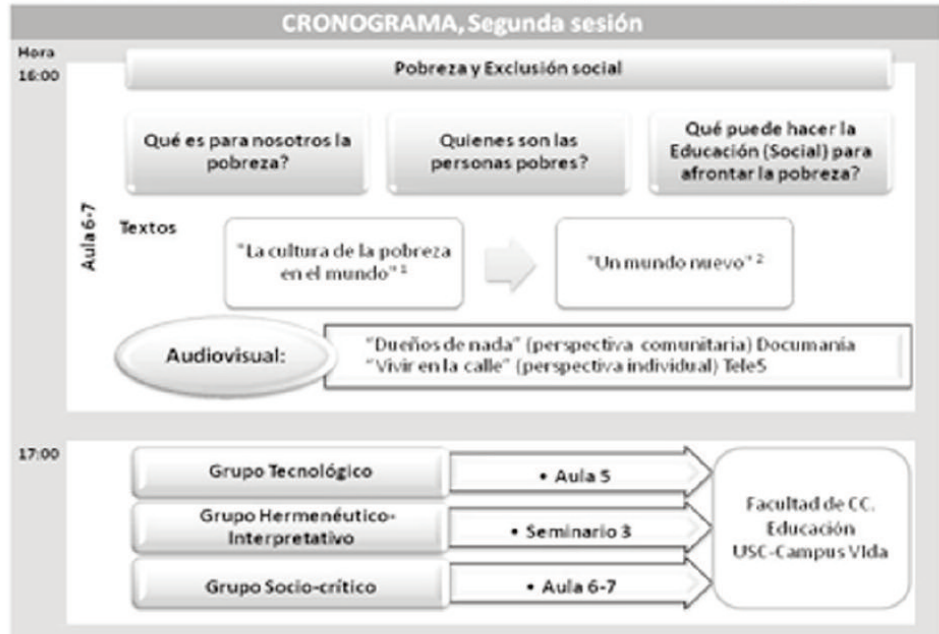

Simulación "Educación Social, desarrollo y pobreza" - Pedagogia Social CRONOGRAMA, Tercera sesión

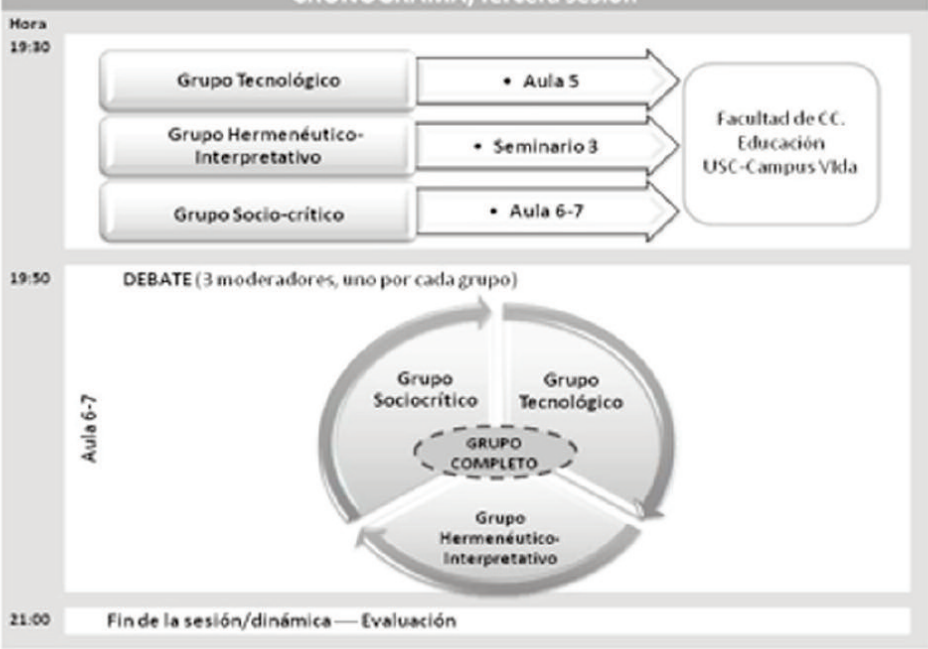

Figura 2. Cronograma y secuenciación de la simulación

Figura 3. Cronograma y secuenciación de la simulación

Figura 4. Cronograma y secuenciación de la simulación 
En la primera sesión -además de explicar la propia dinámica- se hará una breve introducción a los modelos de racionalidad teórica, incidiendo en los postulados esenciales de cada uno de ellos, así como a los tópico que define el tema objeto de análisis, teniendo como eje transversal las "teorías" en la Pedagogía-Educación Social: los procesos de desarrollo, la pobreza y la exclusión social, los derechos de la ciudadanía, etc.

En esta misma sesión y en las siguientes -como mucho en dos de ellas- se inicia la presentación de los documentos (textos, audiovisuales, etc.) que van ilustrando en sus dimensiones micro y macro-social distintas realidades acerca de los procesos de desarrollo, las desigualdades sociales, la pobreza y la exclusión social. Su presentación en el aula, ante el grupo completo de alumnos y alumnas se irá combinando con reuniones de cada uno de los tres grupos que conforman los modelos paradigmáticos, de modo que se van articulando las sesiones más expositivas con las que tienen un carácter más interactivo y participativo.

En las sesiones de debate que se llevan a cabo en cada grupo y tratando de conducir el debate-diálogo final (a veces dándose situaciones de enfrentamiento dialéctico de cierta intensidad) se irán avanzando las respuestas de cada grupo o modelo paradigmático en función de las cuestiones planteadas: ¿Quiénes son las personas pobres? ¿Qué es la pobreza? ¿Qué se puede y debe hacer para combatir la pobreza? ¿Cómo educar ante y/o en las situaciones de pobreza? En definitiva, tratando de aportar una visión diagnóstica-problematizadora de las realidades objeto de estudio, analizando sus circunstancias desde la mirada de la teoría y de cada una de las teorías en la Pedagogía-Educación Social; y, con una visión reivindicativa y/o alternativa, posicionándose acerca de lo que puede y debe hacerse -políticamente, educativamente, socialmente, etc.- para afrontar sus circunstancias.

La última de las sesiones se articula en torno a dos momentos clave: el primero -con una duración aproximada de treinta minutos- se corresponde con el debate que se realiza en pequeños grupos (en aulas diferenciadas) en función de los modelos paradigmáticos (tecnológico, hermenéutico-interpretativo y socio-crítico) a los que están adscritos los estudiantes, con el fin de dar respuesta a las cuestiones planteadas, aludiendo a los contextos que determina el alumnado en la perspectiva local-global que sugieren los documentos escritos y audiovisuales utilizados en la dinámica. Pasado el tiempo, y una vez establecidas en el seno de cada grupo sus estrategias argumentales, el segundo momento de, al menos, una hora de duración- se centra en el debate colectivo, en el que convergen las tres perspectivas teóricas; siendo los moderadores de cada grupo los encargados de otorgar el turno de palabra a los respectivos portavoces, quienes sucintamente -y en primer lugar- explicarán los posicionamientos clave de su paradigma, en relación a la temática estudiada. De este modo, los diferentes roles comienzan a evidenciarse en el diagnóstico inicial de la problemática planteada así como en las estrategias socioeducativas que llevarían a cabo como pedagogos o educadores para afrontarla. Tras esta primera ronda de intervenciones, se articula un diálogo abierto al conjunto de los participantes (en el que llegan a participar de forma intensa entre el 60 y el $75 \%$ de los estudiantes que están en el aula) que refleja las múltiples formas de interpretar y actuar en la sociedad desde la Pedagogía-Educación Social.

Para evaluar la actividad en sí misma y en el conjunto de las valoraciones que se incorporan a la calificación final de los alumnos y de las alumnas de esta asignatura, se aplican -durante y después del desarrollo de la misma- diferentes técnicas, que permiten observar su evolución como un proceso metodológico de enseñanza-aprendizaje, al tiempo que las actitudes y comportamientos tanto del grupo-clase como del conjunto de los participantes en las dinámicas que se establecen: niveles de implicación y participación, el clima del aula, organización y estrategias para el diálogo que se adoptan en los diferentes grupos, etc. Además, y una vez finalizada la simulación, se distribuye entre todos los participantes un cuestionario-ficha (ver Anexo B) en el que podrán expresar sus valoraciones individuales acerca de la dinámica realizada, incidiendo en los aspectos teóricos, temáticos y metodológicos más relevantes.

Concluiremos, poniendo en palabras de los/as estudiantes -reflejadas en las memorias autoevaluativas que realizan en la materia de Pedagogía Social-algunas de las valoraciones que han venido realizando a propósito de la dinámica de simulación en el aula:

- "El tema 3 (Teoría y Teorías de la Pedagogía-Educación Social: modelos de encuadre y racionalidad teórica), desde mi punto de vista, fue uno de los más interesantes y, a la vez, más complejos. La metodología usada para tratar este tema contribuye en gran medida al aprendizaje e interiorización de los tres paradigmas, ya que mediante la dinámica de grupo, se, consiguió que las diferencias básicas entre los tres paradigmas quedaran bien ubicadas en nuestra mente".

- "En mi opinión, fue una de las actividades más importantes y más productivas. Creo la opinión de que esta fue una de las mejores actividades es compartida con muchos de mis compañeros, y la prueba está en que el día del debate, case la totalidad de los alumnos matriculados en la materia estaban presentes, además de que la participación fue de las más altas”.

- "Me gustaría destacar la dinámica de los paradigmas, creo que fue una semana muy interesante, ya que se trataba de conocer diferentes puntos de vista de la realidad desde la perspectiva teórica que hasta el momento desconocíamos; y lo mejor sin lugar a duda fue el debate que se hizo en clase, en el cual sin querer se entendía y se aprendía de una forma distinta las características de los paradigmas, simplemente con interaccionar con los demás compañeros que tenían un paradigma distinto. Un modo de aprender diferente y muy interesante capaz de suscitar interés de una forma totalmente novedosa desde mi punto de vista”.

- "La simulación llevada a cabo me permitió conocer a fondo estas líneas de pensamiento. Desde mi punto de vista, el hecho de tener que meternos en un papel, hizo que realmente aprendiéramos lo fundamental sobre nuestro respectivo paradigma, pero al ter que debatir en el aula, hizo además que comprendiéramos los otros dos".

En su conjunto, como ya hemos anticipado, coinciden en señalar que tanto desde la perspectiva de la enseñanzaaprendizaje de los contenidos abordados como de la dinámica metodológica desarrollada, ha sido una de las experiencias de su formación inicial más motivadoras y relevantes. En esta apreciación se resume la evaluación que los docentes hacen de la dinámica desde su programación hasta su desarrollo en el conjunto de la asignatura. En este 
sentido, se toma nota de las valoraciones que el alumnado hace de las sesiones una vez concluidas, de las aportaciones y sugerencias que realizan, de la comparación entre los procesos y logros de cada curso y los anteriores, de las propuestas orientadas a mejorar la dinámica en el futuro, etc. En todo caso, se procura que la evaluación de esta actividad se inscriba en un proceso evaluativo integral, congruente con la planificación de la materia y la guía docente-discente de la misma.

\section{Bibliografía}

Arias Aranda, Daniel; Romerosa Martínez, Ma Mercedes; Navarro Paule, Andrés J.; Haro Domínguez, Ma Del Carmen Ortega Egea, $M^{\text {a }}$ Teresa (2008). La simulación como herramienta de aprendizaje para la dirección estratégica. Cuadernos de Estudios Empresariales, 18, pp. 33-49.

Bruner, Jerome S. (1969). Hacia una teoría de la instrucción. México: UTEHA (edición original: Toward a Theory of Instruction. Cambridge Mass: Belkapp Pres.)

Caride, Jose Antonio (2003). El pluralismo teórico como argumento epistemológico en el quehacer pedagógicosocial. Pedagogía Social. Revista Interuniversitaria, 10 (segunda época), pp. 123-159.

García, Félix (coord.) (2012). La educación formal y los procesos de inclusión social. Madrid: Cáritas, serie Documentación Social.
Graeml, Felipe R.; Baena, Verónica; Mihai Yiannaki; Simona (2010). La integración de diferentes campos del conocimiento en juegos de simulación empresarial. Revista de Docencia Universitaria, 8 (2), pp. 29-44, http://redaberta.usc.es/redu/documentos/vol8_n2_c ompleto.pdf. Fecha de consulta, 05.10.2012.

Habermas, Jürgen (1982). Conocimiento e interés. Madrid: Taurus.

Martínez, Miquel (ed.) (2008). Aprendizaje servicio y responsabilidad social de las Universidades. Barcelona: Octaedro-ICE/UB.

Matas, Antonio (2003). Los juegos de simulación como recurso formativo. Una aplicación en Educación Ambiental. Bordón, 55 (2), pp. 281-191.

Medina, Antonio María (2001). Los métodos en la enseñanza universitaria. En García-Valcárcel, Ana (coord.). Didáctica universitaria. Madrid: Editorial La Muralla, pp. 155-198.

Valverde, Jesus (2008). Aprender a pensar históricamente con apoyo de soportes informáticos. Revista Cultura \& Educación, 20 (2), pp. 181-199.

Valverde, Jesus (2010). Aprendizaje de la Historia y Simulación Educativa. Tejuelo: Didáctica de la Lengua y la Literatura, 9, pp. 83-99.

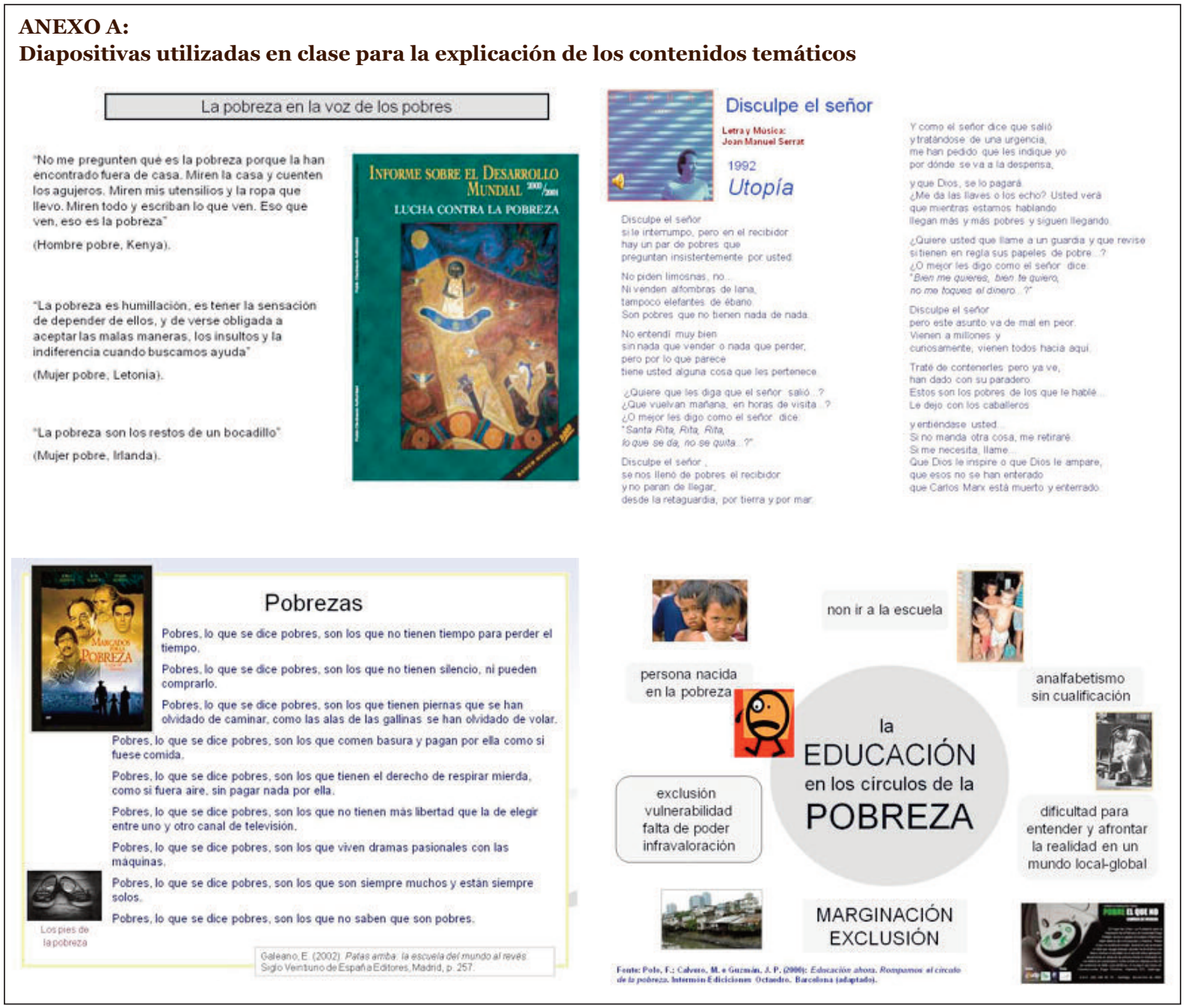




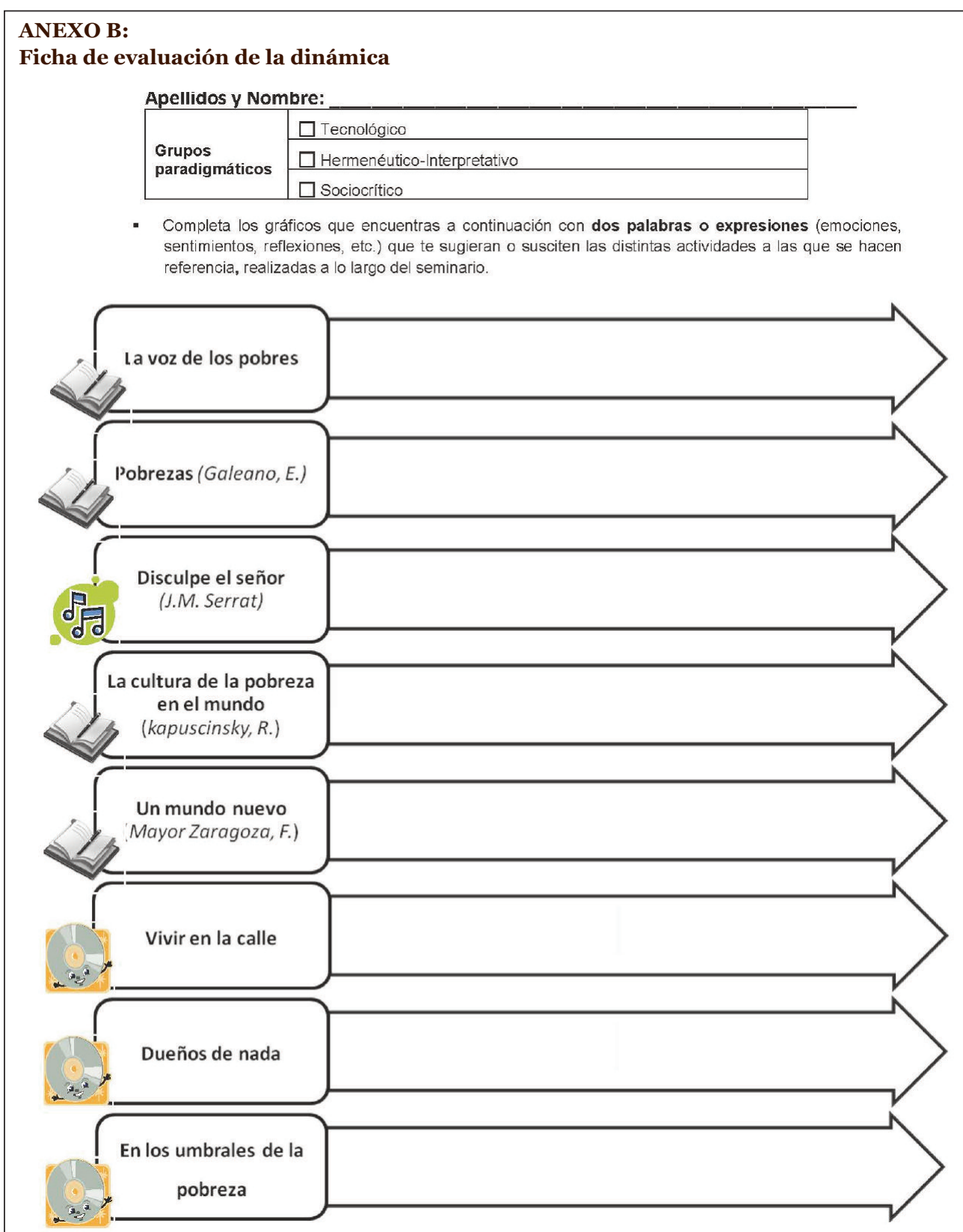

Valora brevemente la temática del seminario y los aprendizajes derivados del mismo para tu formación como educadora:

Valora con brevedad la propuesta metodológica (simulación) en la que se ha sustentado el desarrollo del seminario:

Observaciones y propuestas de mejora que pueden ser tenidas en cuenta de cara al futuro:

Valora en una escala de 1 (mínimo) a 10 (máximo) los siguientes aspectos en relación al seminario:

Interés de las temáticas

Grado de participación del grupo-clase

Innovación metodológico-didáctica

Aprendizajes derivados de la actividad

Valoración global 


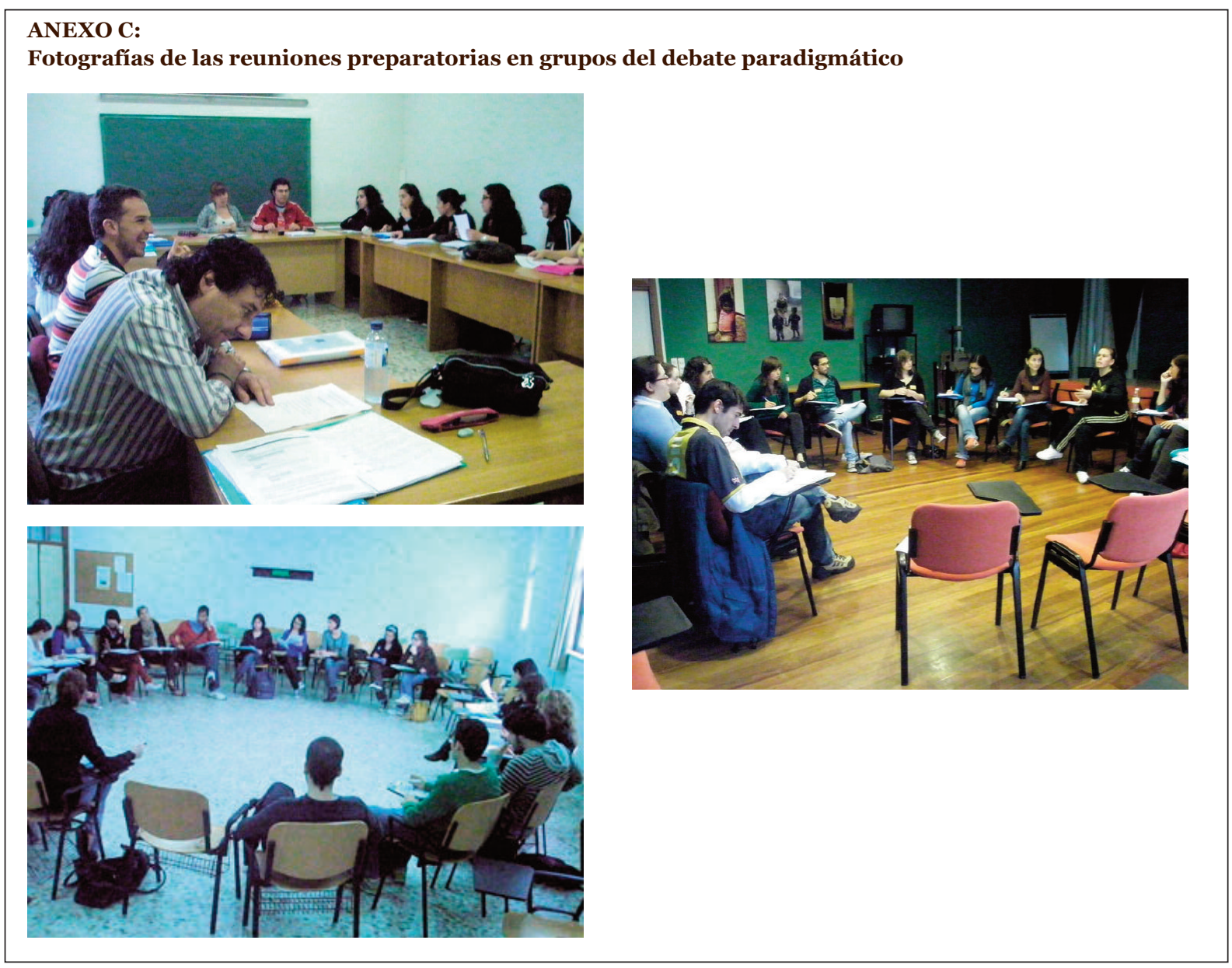

| Cita recomendada de este artículo

Caride Gómez, José Antonio; Gradaíle Pernas, Rita y Teijeiro Bóo, Yésica (2012). El diálogo paradigmático en Educación Social: la simulación como un modo de enseñar y aprender en las aulas universitarias. @tic. revista d'innovació educativa. ( $n^{\circ} 9$ ). URL. Fecha de consulta, $\mathrm{dd} / \mathrm{mm} /$ aaaa. 